\title{
Edema Limbs, CTCAE
}

National Cancer Institute

\section{Source}

National Cancer Institute. Edema Limbs, CT CAE. NCI Thesaurus. Code C143433.

A disorder characterized by swelling due to excessive fluid accumulation in the upper or lower extremities. 